\title{
Reduced and Superreduced Diplatinum Complexes
}

Tania V. Darnton, ${ }^{\mathrm{a}, \sharp}$ Bryan M. Hunter, ${ }^{\mathrm{a}, \ddagger}$ Michael G. Hill, ${ }^{\mathrm{b}}$ Stanislav Záliš, ${ }^{*, c}$ Antonín Vlček Jr., ${ }^{*, c, d}$ and Harry B. Gray*,a

${ }^{a}$ Beckman Institute, California Institute of Technology, Pasadena, CA 91125, United States

${ }^{\mathrm{b}}$ Occidental College, Los Angeles, California 90041, United States

${ }^{\mathrm{c}} \mathrm{J}$. Heyrovský Institute of Physical Chemistry, Czech Academy of Sciences, Dolejškova 3, CZ18223 Prague, Czech Republic

${ }^{\mathrm{d}}$ Queen Mary University of London, School of Biological and Chemical Sciences, Mile End

Road, London E1 4NS, United Kingdom

$\ddagger$ Indicates that these authors contributed equally.

\section{SUPPLEMENTARY INFORMATION}

FIGURES

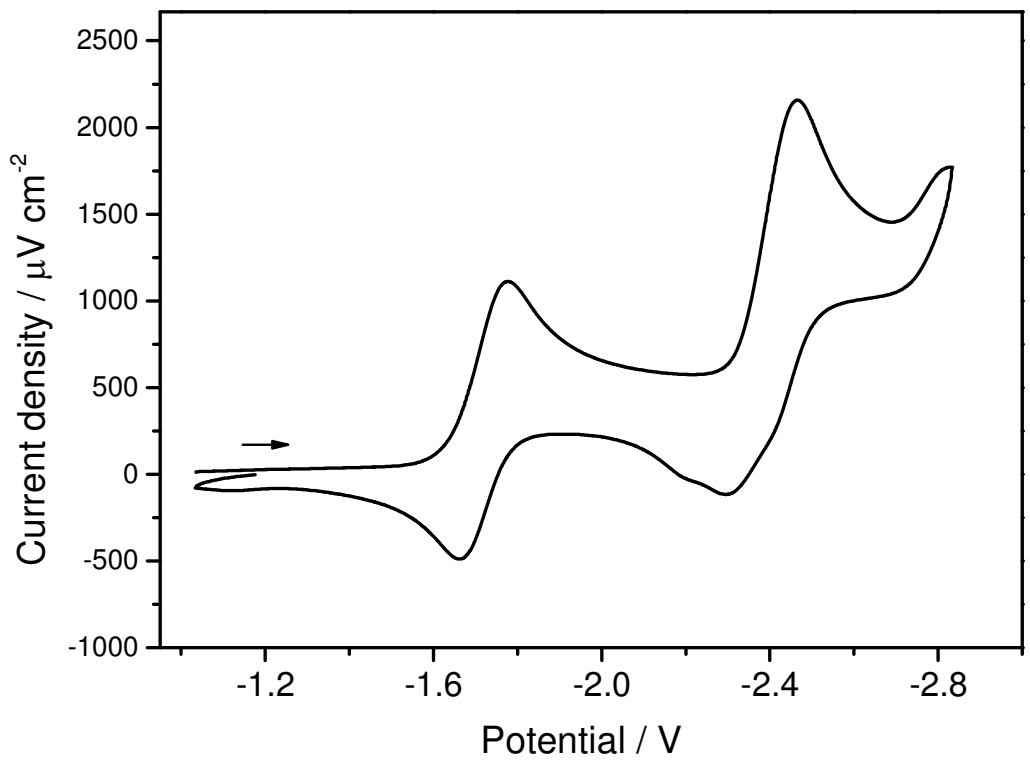

Figure S1. Cyclic voltammogram of $\mathrm{Pt}\left(\mathrm{pop}_{-} \mathrm{BF}_{2}\right)^{4-}$ in $\mathrm{MeCN}$ containing $0.1 \mathrm{M} \mathrm{Bu}_{4} \mathrm{NPF}_{6}$ at room temperature ( $294 \mathrm{~K})$. Potentials vs. $\mathrm{Fc}^{+} / \mathrm{Fc}$. Scan rate $50 \mathrm{mV} / \mathrm{s}$. 


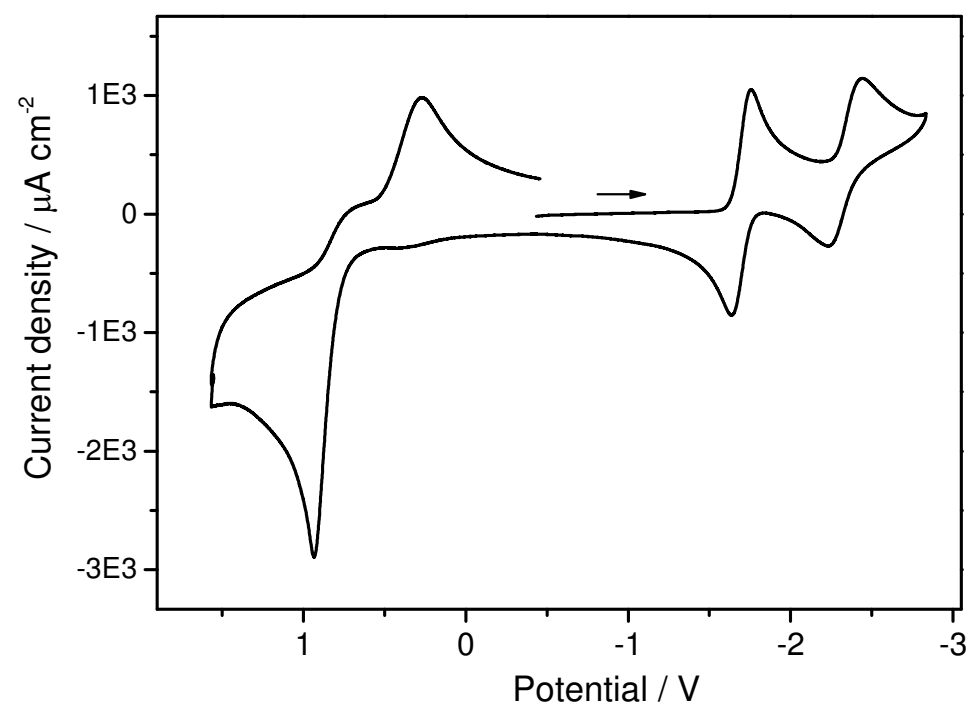

Figure S2. Full-range cyclic voltammogram of Pt(pop-BF $)^{4-}$ in $\mathrm{MeCN}$ containing $0.1 \mathrm{M} \mathrm{Bu}_{4} \mathrm{NPF}_{6}$ at $273 \mathrm{~K}$. Potentials vs. $\mathrm{Fc}^{+} / \mathrm{Fc}$. Scan rate $200 \mathrm{mV} / \mathrm{s}$.
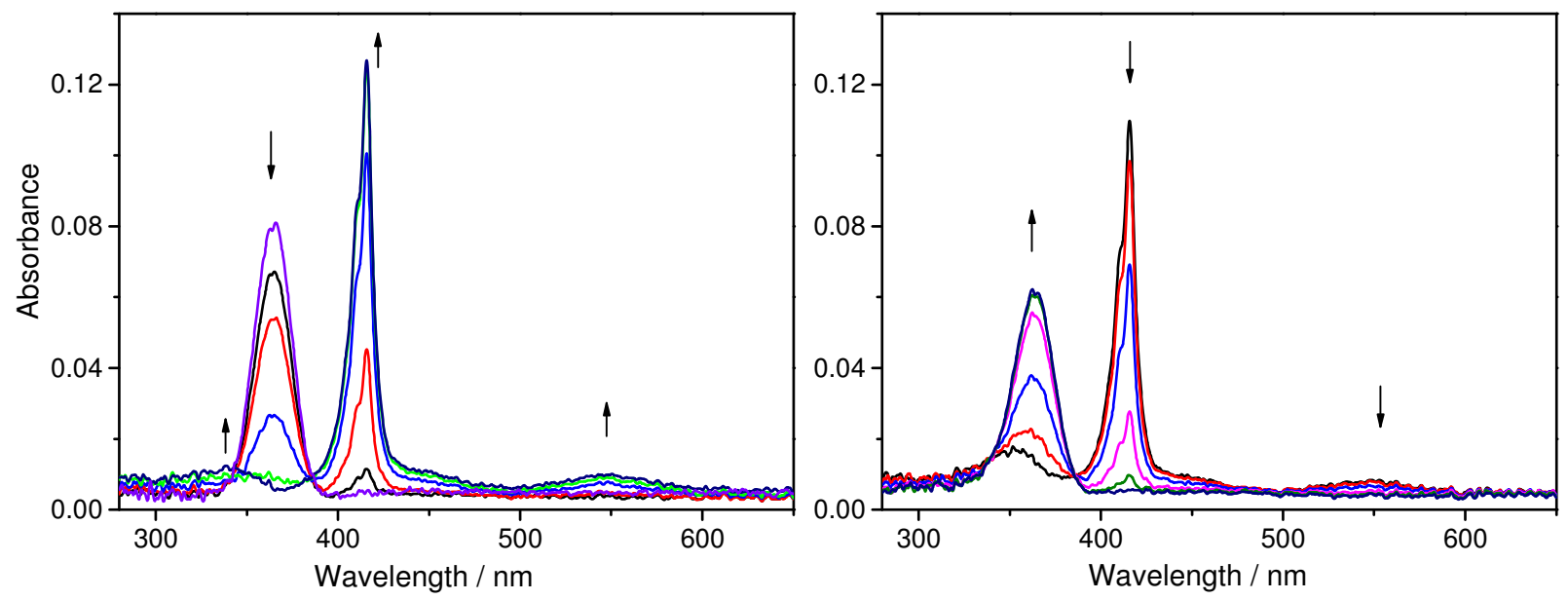

Figure S3. UV-vis absorption spectra monitored in the course of the first $\mathrm{Pt}\left(\mathrm{pop}-\mathrm{BF}_{2}\right)^{4-}$ reduction to $\mathrm{Pt}\left(\mathrm{pop}-\mathrm{BF}_{2}\right)^{5-}$ (left) and subsequent product reoxidation (right). The slightly lower intensities in the right panel are caused by product diffusion out of the spectroscopically probed region. 

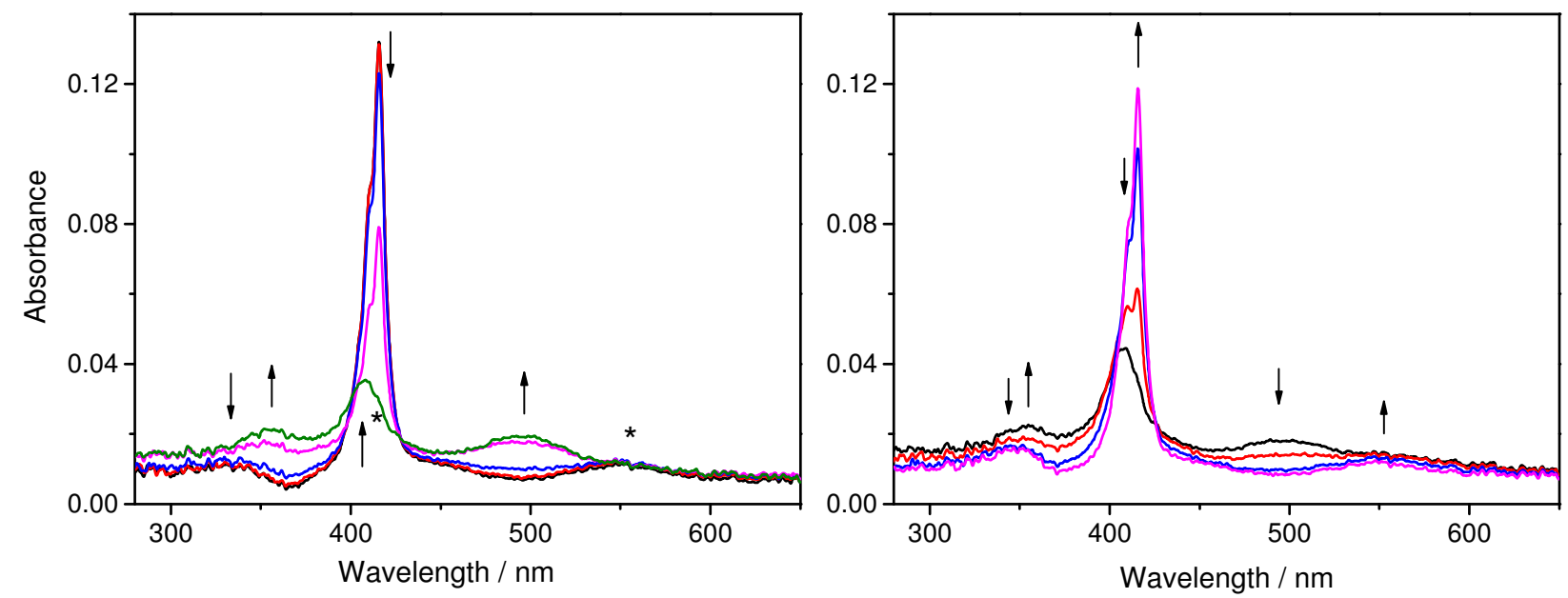

Figure S4. UV-vis absorption spectra monitored in the course of the second $\mathrm{Pt}\left(\mathrm{pop}-\mathrm{BF}_{2}\right)^{4-}$ reduction (left) and subsequent product reoxidation (right). The slightly lower intensities in the right panel are caused by product diffusion out of the spectroscopically probed region. 


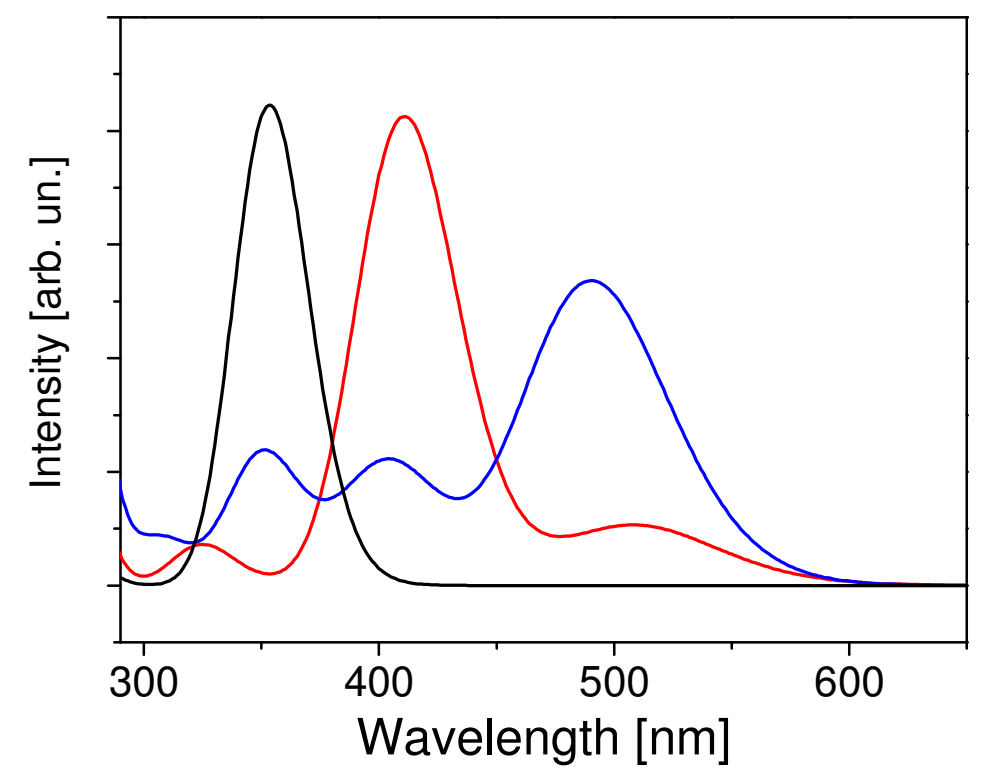

Figure S5a. TD-DFT simulated UV-vis absorption spectra of Pt(pop-BF $)^{n-}(\mathrm{n}=4$ (black), 5 (red) and $6 /$ conformer 6 (blue)). The same FWHM of $3000 \mathrm{~cm}^{-1}$ was assumed for all transitions. TDDFT (PBEO/PCM-MeCN)

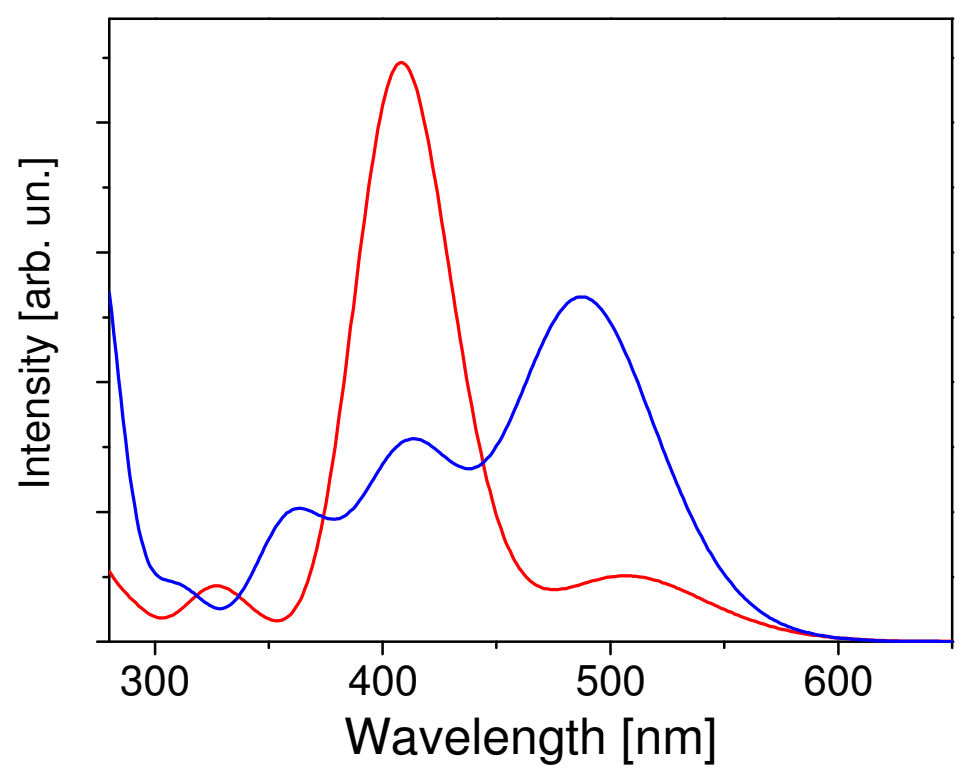

Figure S5b. TD-DFT simulated UV-vis absorption spectra of Pt(pop-BF $)^{n-} \cdot \mathrm{nMe}_{4} \mathrm{~N}^{+}(\mathrm{n}=5$ (red), $6 /$ conformer 6 (blue)). The same FWHM of $3000 \mathrm{~cm}^{-1}$ was assumed for all transitions. TD-DFT (PBEO/PCM-MeCN) 


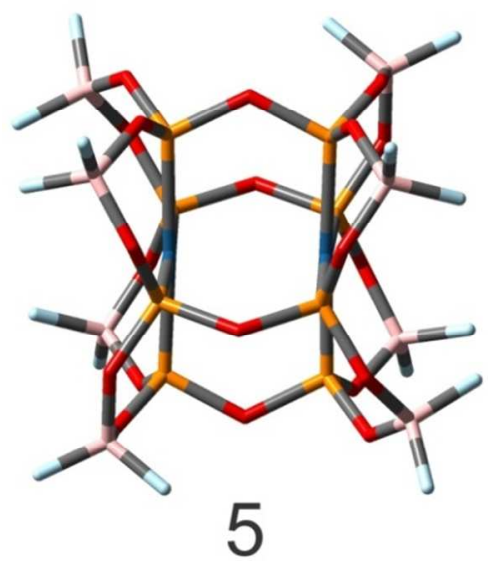

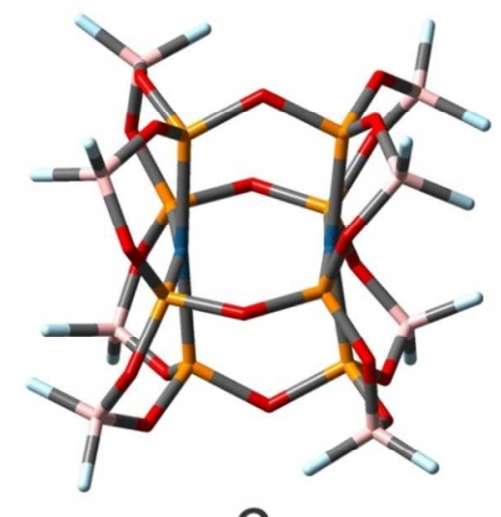

6

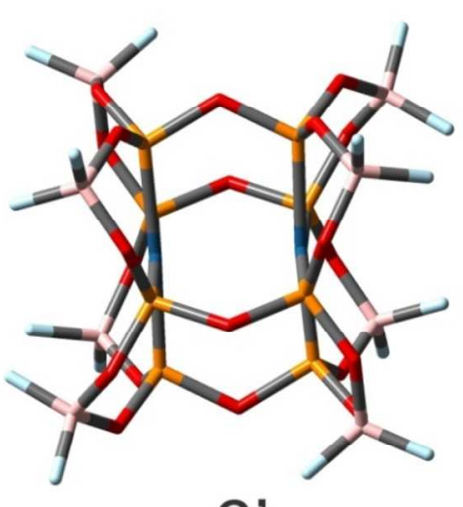

$6^{\prime}$

Figure S6. Left and middle: DFT-optimized structures of Pt(pop-BF $)^{5-}(\mathbf{5})$ and the 6 and $\mathbf{6}^{\prime}$ conformers of Pt(pop-BF $)^{6-}$ in $\mathrm{MeCN}$.

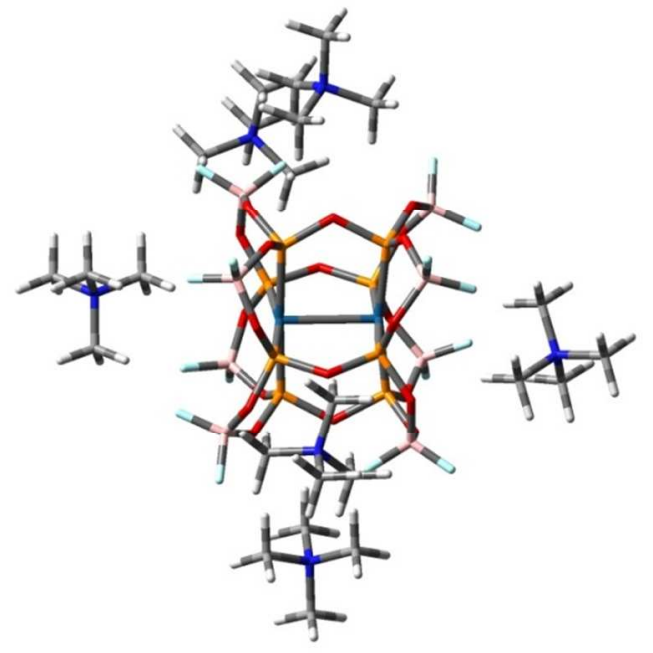

Figure S7. DFT-optimized structure of $\mathrm{Pt}\left(\text { pop- }-\mathrm{BF}_{2}\right)^{6-} \cdot 6 \mathrm{Me}_{4} \mathrm{~N}^{+}$in $\mathrm{MeCN}$. 


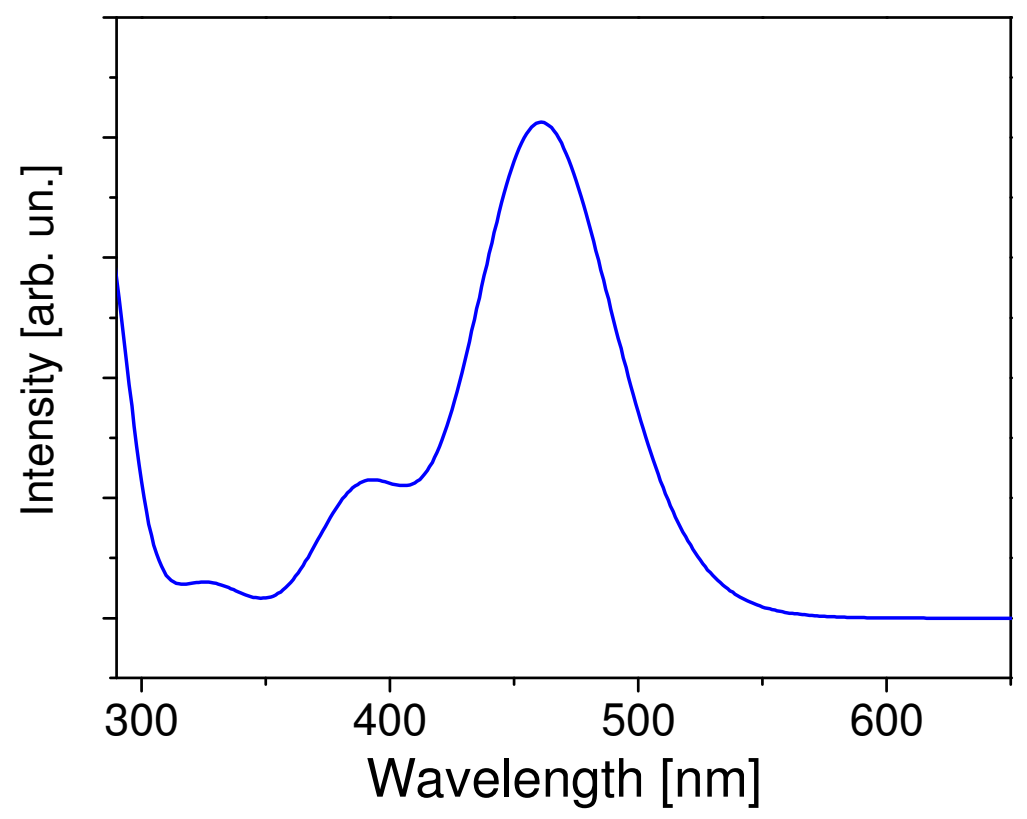

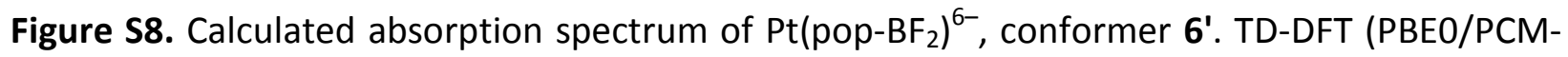
$\mathrm{MeCN})$

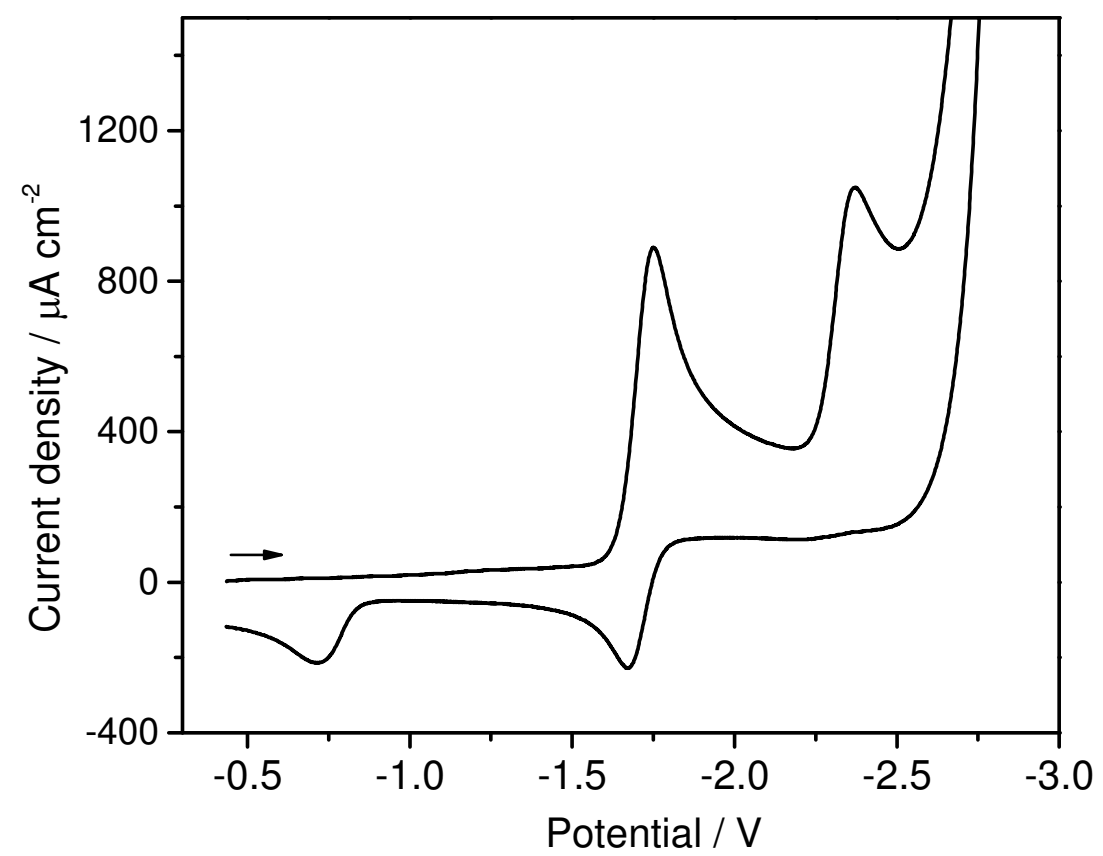

Figure S9. Cyclic voltammogram of Pt(pop-BF $)^{4-}$ in $\mathrm{MeCN}$ containing $0.1 \mathrm{M} \mathrm{Bu}_{4} \mathrm{NPF}_{6}$ and $1 \mathrm{mM}$ $\mathrm{CH}_{2} \mathrm{Cl}_{2}$ at $273 \mathrm{~K}$. Potentials vs. $\mathrm{Fc}^{+} / \mathrm{Fc}$. Scan rate $100 \mathrm{mV} / \mathrm{s}$. 


\section{TABLES}

Table S1. DFT (ADF/PBEO/COSMO-MeCN) calculated spin densities $\rho$ and $g$ and $A(P t)$ (in $\mathrm{MHz}$ ) EPR parameters for $\mathrm{Pt}\left(\mathrm{pop}-\mathrm{BF}_{2}\right)^{5-}$.

\begin{tabular}{lll}
\hline & calc. & exp. \\
\hline$\rho \mathrm{Pt}$ (total) & 0.552 & - \\
$\rho \mathrm{P}$ (total) & 0.389 & - \\
$g_{1}$ & 1.987 & 1.98 \\
$g_{2}$ & 2.035 & 2.03 \\
$g_{3}$ & 2.047 & 2.04 \\
$g_{1}-g_{3}$ & 0.060 & 0.060 \\
$g_{\text {iso }}$ & $2.023^{\mathrm{a}}$ & \\
$\mathrm{A}_{1}(\mathrm{Pt})$ & 599 & 900 \\
$\mathrm{~A}_{2}(\mathrm{Pt})$ & -430 & 550 \\
$\mathrm{~A}_{3}(\mathrm{Pt})$ & -428 & 550 \\
$\mathrm{~A}_{1}\left(\mathrm{Pt} \mathrm{t}^{\prime}\right)$ & 623 & 900 \\
$\mathrm{~A}_{2}\left(\mathrm{Pt} \mathrm{t}^{\prime}\right)$ & -419 & 500 \\
$\mathrm{~A}_{3}\left(\mathrm{Pt} \mathrm{t}^{\prime}\right)$ & -420 & 350 \\
\hline \multicolumn{2}{c}{${ }^{\mathrm{a}}$ Calculated as $g_{\text {iso }}=\left(\left(g_{1}{ }^{2}+g_{2}{ }^{2}+g_{3}{ }^{2}\right) / 3\right)^{1 / 2}}$.
\end{tabular}


Table S2. DFT-calculated (PBEO/PCM-MeCN) structural parameters of Pt(pop-BF $)^{5-}$ and the two conformers of $\mathrm{Pt}\left(\mathrm{pop}-\mathrm{BF}_{2}\right)^{6-}$. Atom $\mathrm{P} 5$ is in alignment with atom $\mathrm{P} 1$, etc.

\begin{tabular}{|l|c|c|c|}
\hline Bond & $\mathrm{n}=5$ & $\mathrm{n}=6 /$ conf 6 & $\mathrm{n}=6 /$ conf $^{\prime}$ \\
\hline Pt1-Pt2 & 2.803 & 2.739 & 2.745 \\
\hline Pt-P1 & 2.277 & 2.268 & 2.255 \\
\hline Pt-P2 & 2.277 & 2.258 & 2.255 \\
\hline Pt-P3 & 2.278 & 2.265 & 2.257 \\
\hline Pt-P4 & 2.278 & 2.255 & 2.257 \\
\hline Pt-P5 & 2.277 & 2.256 & 2.256 \\
\hline Pt-P6 & 2.277 & 2.260 & 2.255 \\
\hline Pt-P7 & 2.278 & 2.260 & 2.255 \\
\hline Pt-P8 & 2.278 & 2.266 & 2.256 \\
\hline P-O(-P) (average) & 1.634 & 1.644 & 1.645 \\
\hline angle & & & \\
\hline Pt2-Pt1-P1 & 91.9 & 87.3 & 93.1 \\
\hline Pt2-Pt1-P2 & 91.9 & 96.2 & 91.7 \\
\hline Pt2-Pt1-P3 & 90.8 & 87.5 & 91.7 \\
\hline Pt2-Pt1-P4 & 90.8 & 96.3 & 93.1 \\
\hline Pt2-Pt1-P5 & 91.9 & 97.1 & 92.3 \\
\hline Pt2-Pt1-P6 & 91.9 & 88.4 & 92.3 \\
\hline Pt2-Pt1-P7 & 90.8 & 96.8 & 90.8 \\
\hline Pt2-Pt1-P8 & 90.8 & 88.3 & 90.8 \\
\hline
\end{tabular}

Table S3. TD-DFT (PBEO/PCM-MeCN) calculated lowest singlet excitation energies (eV) for $\mathrm{Pt}\left(\mathrm{pop}-\mathrm{BF}_{2}\right)^{6-} /$ conformer $\mathbf{6}^{\prime}$ with oscillator strength larger than 0.003 .

\begin{tabular}{|c|c|c|c|c|}
\hline State & $\begin{array}{c}\text { Main contributing excitations } \\
(\%)\end{array}$ & $\begin{array}{c}\text { Transition energy } \\
\mathrm{eV}(\mathrm{nm})\end{array}$ & $\begin{array}{c}\text { Oscillator } \\
\text { strength }\end{array}$ & $\begin{array}{c}\text { Exptl. } \\
\mathrm{eV}(\mathrm{nm})\end{array}$ \\
\hline $\mathrm{b}^{1} \mathrm{~A}$ & $95(\mathrm{HOMO} \rightarrow$ LUMO) & $2.69(461)$ & 0.284 & 461 \\
\hline $\mathrm{c}^{1} \mathrm{~A}$ & $93(\mathrm{HOMO} \rightarrow \mathrm{LUMO}+4)$ & $3.18(404)$ & 0.076 & 490 \\
\hline $\mathrm{d}^{1} \mathrm{~A}$ & $99(\mathrm{HOMO} \rightarrow$ LUMO+6) & $3.75(331)$ & 0.009 & 356 \\
\hline $\mathrm{e}^{1} \mathrm{~A}$ & $98(\mathrm{HOMO} \rightarrow$ LUMO+7) & $3.84(323)$ & 0.012 & \\
\hline
\end{tabular}

